\title{
\#Metoo, la movilización digital en México: respaldo, crítica y oposición en la esfera civil
}

\section{\#Metoo, digital mobilization in Mexico: Support, criticism and opposition in the Civil Sphere}

\author{
Luz Ángela Cardona Acuña• \\ Nelson Arteaga Botello*•
}

\section{Resumen}

Se analiza la controversia en los medios de comunicación mexicanos por la supuesta relación del movimiento \#Metoo con el suicidio de un músico acusado de acoso. Esta controversia abrió un debate sobre la pertinencia de la denuncia anónima del acoso, sus implicaciones legales, sociales y personales, tanto para las víctimas como para los acusados. Siguiendo la propuesta teórica y metodológica de la sociología cultural, se revisaron las columnas de opinión que trataron el tema. El movimiento fue interpretado en los medios como: a) una expresión de justicia legítima; b) una manifestación justificada, pero contaminada por el rencor $y$ la venganza, $y$ c) como una expresión que buscó desde un principio el linchamiento digital de presuntos acosadores. Esta disputa permite comprender los desafíos de la justicia feminista ante casos de violencia contra la mujer.

Palabras clave: movilización feminista, Metoo, esfera civil, acoso, justicia.

\begin{abstract}
The controversy in the Mexican media is analyzed for the alleged relationship of the \#Metoo movement with the suicide of a musician accused of harassment. This controversy opened a debate on the relevance of the anonymous report of the harassment, its legal, social and personal implications, both for the victims and for the accused. Following the theoretical-methodological proposal of cultural sociology, a review was made of the opinion columns that dealt with the subject.The movement was interpreted in the media as: a) an expression of legitimate justice; $b$ ) a justified manifestation but contaminated by rancor and revenge; $c$ ) as an expression that sought from the beginning the digital lynching of alleged harassers. This dispute allows us to understand the challenges of feminist justice in cases of violence against women.
\end{abstract}

Keywords: feminist mobilization, Metoo, civil sphere, sexual harassment, justice.

-Doctora en Investigación en Ciencias Sociales, Facultad Latinoamericana de Ciencias Sociales, sede México. Profesora-investigadora adscrita al Centro de Estudios e Investigaciones Interdisciplinarias, Universidad Autónoma de Coahuila. ORCID: 0000-000 I-8I73-7466.

- Doctor en Sociología por la Universidad de Alicante. Profesor-investigador adscrito a la Facultad Latinoamericana de Ciencias Sociales, sede México. Correo electrónico: nelson.arteaga@flacso.edu. mx. ORCID: 0000-0002-2660-7877.

Fecha de recepción: 28 de julio de 2020. Fecha de aceptación: 21 de enero de 2021. 


\section{Introducción}

En este artículo se examina la disputa en torno a la legitimidad del movimiento \#Metoo para demandar la sanción, resarcimiento y desagravio de las mujeres frente al acoso en el caso mexicano. La disputa se desató frente al suicidio del músico Vega-Gil tras rechazar las acusaciones de acoso en su contra. Este evento generó un debate en prensa que reflejó una competencia entre discursos y narrativas a partir de códigos binarios con los que se apoyó o no al \#Metoo. Quienes defendieron al \#Metoo lo calificaron como puro y juzgaron que las personas que administraban la cuenta y las denunciantes eran activistas y víctimas conscientes, informadas, autónomas y críticas, impulsadas por el deseo de cambiar las cosas - en otras palabras, actores legítimamente civiles-. Quienes criticaron al movimiento valorándolo como impuro, señalaron que, aunque se justificaba, su espíritu se había contaminado por mujeres atrapadas por el rencor y la venganza y no buscaban en ningún momento activar las estructuras y las instituciones de justicia. Finalmente, hubo quienes se opusieron al \#Metoo — pese a la necesidad de actuar contra el acoso — señalando que sus promotoras estaban impulsadas por valores anticiviles, es decir, eran irracionales, imprudentes e incapaces de autocrítica.

Este tipo de análisis es relevante porque permite comprender cómo la disputa sobre la supuesta responsabilidad del \#Metoo en la muerte del músico, desató una controversia en los medios de comunicación sobre la pertinencia de un movimiento de denuncia anónima del acoso - la responsabilidad jurídica y social de las denuncias-y sobre las implicaciones legales, sociales y personales que tiene para los acusados. Estas controversias que pueden encontrarse en otros contextos nacionales cuando hombres - conocidos públicamente- se han quitado la vida al enfrentar acusaciones de acoso. El suicidio de docentes universitarios 
a nivel global, ${ }^{1}$ músicos, directores de museos, ${ }^{2}$ actores, ${ }^{3}$ desarrolladores de videojuegos, ${ }^{4}$ así como escritores y cantantes, ${ }^{5}$ imprime un acento particular al debate sobre el sentido de la denuncia anónima a través de redes sociales, particularmente Twitter, así como juicios sobre el carácter inculpatorio o exculpatorio de su acción. ${ }^{6}$

Siguiendo la propuesta metodológica de la sociología cultural, se analizó el debate en las instituciones comunicativas de la esfera civil, particularmente en la prensa. Se revisaron las 41 columnas publicadas sobre el tema en los diarios Excélsior, El Universal, Milenio, Reforma y La Jornada, entre el 21 de marzo y el 17 de abril de 2019 (periodo en el que se publicó el mayor número de columnas sobre el tema). En la medida en que la crónica periodística es una interpretación sobre los acontecimientos, se revisaron diferentes fuentes y líneas editoriales a efectos de contar con la mayor cantidad de evidencia empírica de la disputa (Ortiz et al., 2005: 406; McCarthy et al., 1996: 479). Las columnas periodísticas son parciales y sesgadas en las interpretacio-

\footnotetext{
I. Al respecto está el caso de un profesor de literatura en Pakistán; véase: https:// www.pakistantoday.com.pk/2019//0/19/false-allegation-of-harassment-forces-maocollege-lecturer-to-kill-self/)

2. Como sucedió con la denuncia al director de un museo en Suecia; véase https://cronicaglobal.elespanol.com/cronica-directo/sucesos/cantanteopera-culpa-metoo-muerte-marido_I584 I4_I02.html; https://www.spikedonline.com/2018/08/03/the-metoo-suicides/; https://www.breitbart.com/ europe/20I8/03/2I/swedish-theatre-director-commits-suicide-false-metoomovement-allegations/)

3. Es el caso de un actor coreano; véase: https://www.chiangraitimes.com/ thailand-national-news/world-news/famed-south-korean-actor-commits-suicideafter-alleged-sexual-abuse-by-metoo-movement/)

4. Al respecto véase: https://www.wired.com/story/videogames-industry-metoomoment-male-fragility/

5. Como sucedió con un escritor de Saltillo; véase: https://www.elnorte.com/ se-suicida-escritor-saltillense-acusado-en-metoo/ar I698165? referer=--7d61616 5662f3a3a6262623b727a7a7279703b767a78--)

6. Algunos otros casos que involucran adolescentes o funcionario locales están en las siguientes ligas: https://thefederalist.com/2019/09/24/when-men-kill-themselvesover-unproven-allegations-me-too-has-gone-too-far/ y https://thefederal.com/ states/north/delhi/delhi-teen-commits-suicide-after-being-tagged-in-metoo-post/
} 
nes que ofrecen, debido a que se construyen en marcos de interpretación anclados en referentes morales específicos (Earl et al., 2004: 67; Río, 2008: 60).

Este sesgo es relevante porque los medios de comunicación son una fuente para observar cómo las interpretaciones de los hechos activan la discusión en la esfera civil. Como sostienen Butler y Luengo (2016: 284), los medios de comunicación construyen mensajes que traducen situaciones concretas en códigos civiles universales a través de evaluaciones y narraciones, al tiempo que — siguiendo a Gupta (2015: 193) — sus discursos narrativizan la vida cotidiana e imaginan colectividades. Como explica Alexander (2006), las columnas de opinión de los periódicos dan cuenta de los motivos, relaciones e instituciones, contaminantes o purificadoras, conectando los presupuestos de la esfera civil. En cada referencia periodística se analizó qué tipo de motivos, relaciones e instituciones se imputaban al \#Metoo y su relación con el suicidio de Vega-Gil.

Como sugieren Arteaga y Arzuaga (2017), las disputas por el carácter civil o anticivil que se imputan distintos actores deben ser evaluadas a partir de las siguientes preguntas: ¿qué motivos se imputan entre sí? ¿Qué relaciones se atribuyen? Y ¿a qué tipo de instituciones se acusan de pertenecer? Primero, se analizó cómo cada posición definió a los actores: ya sea como racionales, razonables, realistas y objetivos, o por el contrario como irracionales, prejuiciosos, irrealistas y subjetivos. Segundo, se analizó si cada posición evaluó a los actores como la expresión de mujeres envueltas en relaciones abiertas, críticas y francas —es decir plenas de virtudes civiles - o cerradas y discrecionales, orientadas a beneficiar a un sector particular de la sociedad. Tercero, se registró el tipo de instituciones que supuestamente defendían: reguladas, bajo el imperio de la ley, equitativas e inclusivas o, por el contrario, arbitrarias, discrecionales, jerárquicas y excluyentes. Finalmente, siguiendo a Alexan- 
der (2008) y a Geertz (2003), se procedió al análisis de la estructura binaria en la disputa, así como de los hechos que fueron evocados por los columnistas al analizar el suicidio de Vega-Gil, todo lo cual permitió reconstruir el contexto en el que se asentaron las interpretaciones sobre el \#Metoo. Se hace referencia a los argumentos y a las imputaciones que desató \#Metoo en la esfera civil para analizar la densidad del debate sobre el mismo. Las citas empleadas dan cuenta de las diferentes voces e imputaciones. Con esta forma expositiva se pretende consolidar una sólida base empírica con la que se muestra cómo las atribuciones civiles e anticivil al \#Metoo operan y se expresan en las distintas columnas de opinión, independientemente de la línea editorial del periódico. $^{7}$

Los trabajos que analizan las denuncias a través de \#Metoo en redes sociales se han orientado a enfatizar su capacidad para dar voz a las mujeres que han sido acosadas y exigir la reparación del daño provocado por sus acosadores (Banet-Weiser, Gill y Rottenberg, 2019; De Benedictis, Orgad y Rottenberg, 2019; Johansson y Scaramuzzino, 2019; Hasunuma y Ki-young, 2019; Clark-Parsons, 2019; Allison, 2019). Otras investigaciones consideran las denuncias anónimas a través de Twitter como un mecanismo de empoderamiento, pero advierten que es necesario reforzar los aparatos de justicia para garantizar la aplicación de la ley, porque la sola denuncia no garantiza cambios a largo plazo en las estructuras de dominación (McKinney, 2019; Lamas, 2018; Di Bennardo, 2019; Mendes, Keller y Ringrose, 2019; Giliespie, 2019). Finalmente, un tercer conjunto de trabajos — apenas incipiente- ha considerado

7. Esta propuesta metodológica estaba basada en el concepto "etnología de la prensa", que implica reproducir las voces de aquellos que opinan en ella de la misma manera que el etnógrafo recoge las voces de los informantes clave en las comunidades en tanto expresiones de sus mundos de vida, cosmogonías de sentido y formas de estructuradas de entender ciertos problemas y sus soluciones. Una reflexión al respecto se puede encontrar en Augé (2010). 
el suicidio de los presuntos acosadores; sin embargo, se concentran en advertir que estos acontecimientos generan respuestas a favor y en contra del \#Metoo, sin reconstruir narrativa y discursivamente cómo se construye esta disputa (Lilja y Johansson, 2018; Sosa, 2019; Rivera, 2020). Con este trabajo se busca contribuir precisamente a la comprensión de las dinámicas de construcción narrativa y discursiva sobre la disputa frente al \#Metoo en la esfera civil en el caso particular de México a partir de un evento específico, el suicidio de Vega-Gil.

\section{Conceptos para el análisis}

La sociedad genera discursos, narrativas e instituciones que producen al mismo tiempo crítica y solidaridad, así como inclusión y exclusión social. Éstos logran concebir culturalmente un cierto tipo de comunidad universalizante que suele traducirse en instituciones (Alexander y Tognato, 2018). Alexander (2006) denomina a este mundo de valores e instituciones como la esfera civil. Una esfera que produce una solidaridad distinta a la que se puede encontrar en la economía, la familia, la religión y el Estado (Alexander, 2015). Los compromisos de solidaridad de la esfera civil no se cumplen nunca plenamente debido a que hay un constante cuestionamiento sobre la capacidad de integración e inclusión de la sociedad. Lo que explica que siempre existan distintas interpretaciones que buscan ampliar las bases de la inclusión.

Las interpretaciones y críticas sobre el problema de la inclusión tienen lugar en las instituciones comunicativas y regulativas de la esfera civil. Las primeras, reflejan y difunden — como sugieren Kivisto y Scorttino (2015) — las posturas, pasiones e intereses de quienes se asumen como parte de una sociedad o forman una red de actores que 
hablan en nombre de la sociedad, hacia la sociedad y como sociedad. Entre estas instituciones están los medios de comunicación, las asociaciones voluntarias y los movimientos sociales. Las instituciones regulativas son aquellas que ante el reclamo social de solidaridad poseen el derecho a tomar decisiones vinculantes — como sucede, por ejemplo, con los cargos electivos y tribunales.

Los movimientos sociales son considerados desde la teoría de la esfera civil como mecanismos que permiten traducir las disputas sobre la inclusión, la solidaridad y la reparación civil (Alexander, 2006; Eyerman y Jamison, 1990). Los movimientos pueden recibir muestras de apoyo y soporte, así como respuestas en contra que apelan a mantener la interpretación normalizada de las formas de solidaridad y sus instituciones. Estas disputas se dirimen en la esfera civil a través de discursos en que mutuamente se califican a los actores que participan en ellas como puros o impuros (Douglas, 1979), legítimos o ilegítimos, que merecen ser incluidos o excluidos del debate público o de la sociedad (Alexander, 2006). En las sociedades democráticas estas atribuciones se hacen por medio de un código civil que permite a ciertos actores asumirse con virtudes cívicas y juzgar a los otros como condensaciones del vicio cívico. La calificación e identificación con lo puro y lo impuro es determinante para la concreción del sentimiento de solidaridad, la apatía, la descripción y la prescripción propios de la esfera civil.

Los movimientos feministas han buscado transformar las estructuras de dominación impuestas por el sistema heteropatriarcal, el cual se sostiene en valores y principios no civiles anclados en modelos tradicionales de familia, organización del trabajo y convivencia en la vida pública (Alexander, 2017). Los movimientos feministas se han caracterizado por ejercer una presión a favor de la igualdad de género y la producción de mecanismos de reparación civil para resarcir y eliminar la dominación, discriminación y violencia contra 
las mujeres (Luengo, 2018). La primera y la segunda ola del feminismo permitieron cambios en las estructuras del poder, ampliaron los márgenes de participación ciudadana de las mujeres y permitieron un cambio cultural sobre el sentido de lo femenino (Alexander y Mast, 2011). Estas demandas han despertado la oposición de quienes consideran que el feminismo mina valores juzgados como esenciales para la vida en sociedad: la familia tradicional, los roles asignados a hombres y mujeres, así como otros principios morales no civiles que garantizan una integración y solidaridad social excluyente para las mujeres.

Uno de los logros más importantes de los movimientos feministas es que permitieron a las mujeres incorporarse en el mundo del trabajo fuera de lo doméstico (Jonge, 2018; Perrot, 2019). Desafortunadamente, en este espacio las estructuras objetivas y subjetivas de orden patriarcal persisten (McLaughlin, Uggen y Blackstone, 2012). Esto puede observarse en la asimetría salarial que favorece a los hombres, así como en la prioridad que se da a éstos en los ascensos laborales (Acker, 2006; Basu, 2003). Además, las mujeres enfrentan insinuaciones sexuales a modo de coerción y acoso; prácticas que se han considerado por mucho tiempo como propias, normales y rutinarias (Holland y Cortina, 2013; McLaughlin, Uggen y Blackstone, 2012). Esto ha favorecido que la agresividad, el machismo, la autonomía y la superioridad masculina se consideren como valores positivos; de igual forma la sumisión, la deferencia y la dependencia femenina frente a los principios y códigos de la masculinidad (Mueller, Mulinge y Glass, 2002).

En los últimos años se ha dado un cambio en estos códigos (Folbre, 2020; O’Brien, 2020; Laguna, 2020; Bowman, 2020). Como sugiere Alexander (2019), la agresividad sexual masculina se ve con más frecuencia con desconfianza, como una expresión deshonesta y perversa; es decir, como impura o anticivil. Mientras que sus víctimas comienzan cada vez 
más a ser valoradas como confiables, honestas y civiles (Clark-Parsons, 2019). Esto ha permitido la emergencia de nuevos protagonistas y antagonistas en una narrativa de liberación acerca de combatir la opresión y crear justicia en los espacios de trabajo, que favorece a las mujeres (Alexander, 2019: 90). Si bien esa narrativa no se ha consolidado del todo, es cierto que con mayor frecuencia los hombres en el mundo del trabajo dejan de ser admirados por sus pretensiones sexuales, para ser vistos como perpetradores - es decir, como actores anticiviles e impuros-. Por otro lado, las mujeres poco a poco pasan de ser consideradas como víctimas, para convertirse en heroínas que cuentan sus historias de resistencia y lucha - actores puros o civiles- que transforman las estructuras de dominación heteropatriarcal (Hearn, 2018; Hebert, 2018).

El \#Metoo en Estados Unidos puso en la palestra mediática global el problema del acoso en los espacios laborales. Su acción permitió incluso reforzar reformas legales para sancionar esa práctica no sólo en Estados Unidos, sino en otros países (Rhode, 2019). El \#Metoo fue respaldado por movimientos antiacoso que venían ya trabajando en otros países, como \#BalanceTonPorc en Francia, \#QuellaVoltaChe en Italia y \#YoTambién en España y América Latina (Peña et al., 2020). ${ }^{8}$ Pero al tiempo que recibía apoyos, también fue objeto de críticas. Un grupo de actrices francesas —encabezada por Catherine Deneuve- bajo el título La otra palabra, cuestionó el supuesto puritanismo del \#Metoo y promovió defender la libertad de importunar entre hom-

8. En octubre de 2017 Alyssa Milano popularizó el hashtag \#Metoo. Milano denunció el acoso sexual que sufrió del productor de Hollywood HarveyWeinstein, señalando: "Si todas las mujeres que han sido acosadas o agredidas sexualmente hicieran un tuit con el \#Metoo, podríamos mostrar a la gente la magnitud del problema" (D'Zurilla, 2017). Su mensaje fue reproducido más de medio millón de veces y en Facebook fue usado por 4.7 millones de personas (Ohlheiser, 2017). Actrices, académicas y activistas respaldaron el hashtag (Ushma, 20I7; Cadei, 20I7), desatando una oleada de denuncias en redes sociales en Estados Unidos, algunos países de Europa, Asía, África y América Latina (Hasunuma y Ki-young, 2019). 
bres y mujeres (Arteaga y Cardona, 2020). Cuando \#Metoo llegó a México en aquel momento, no generó demasiado eco pese a que el acoso es un grave problema para millones de mujeres en el país (Frías, 2020; Sánchez, 2020; McLean, 2020; Parreno et al., 2018). ${ }^{9}$ No obstante, en marzo de 2019 se registraron 2,000 denuncias en redes sociales bajo el hashtag \#Metoo (Nicolas et al., 2019). El fenómeno desató una disputa en medios de comunicación, lo que generó dos interpretaciones en competencia en términos de la lógica del discurso binario de la esfera civil.

La primera interpretación justificó la denuncia anónima como una vía adecuada para exigir la reparación del daño ocasionado por el acoso. Se valoró como un medio para visibilizar el problema frente a la incapacidad de las instituciones para garantizar la verdad, la justicia y la no repetición contra las víctimas. Algunas personas que apoyaron el movimiento también consideraron que éste rápidamente se contaminó con denuncias falsas o mal intencionadas de mujeres que buscaban desprestigiar a figuras públicas masculinas. Otro grupo de articulistas justificó la molestia y el hartazgo de las mujeres frente al acoso, aunque calificó como inadecuado el mecanismo de denuncia —anónimo, sin pruebas y asumiendo de facto la culpabilidad de los acusados-. Se interpretó que el \#Metoo estaba contaminado desde el principio, ya que era juez y parte en las acusaciones.

9. Según la Encuesta Nacional sobre la Dinámica de las Relaciones en los Hogares (2016), 19.2 millones de mujeres fueron sometidas en algún momento de su vida a algún tipo de intimidación, hostigamiento, acoso o abuso sexual. Sin embargo, los datos de esta encuesta no permiten apreciar la dinámica del acoso en el espacio laboral fuera de casa. No obstante, la información proporcionada por la administración pública federal sobre los casos de acoso en el gobierno es un buen referente para entender la dimensión del problema. Según datos públicos, en 2017 se registraron 145 denuncias entre los funcionarios de la administración pública federal por presuntos casos de hostigamiento o acoso sexual (Inmujeres, 2017: 13). En el año 2018 la cifra fue de 21 I denuncias por los mismos motivos (Inmujeres; SFP, Conavim, 2018: I5). 
El suicidio de Vega-Gil sumó a esta disputa un elemento más: la supuesta responsabilidad del \#Metoo por la muerte del músico. Como sugiere Eyerman (2011), los actos de violencia - entre ellos los suicidios - propician debates en la esfera civil en los que se señala directa o indirectamente a los posibles responsables. Además, se definen causas sociales y se sugieren las dinámicas que lo han hecho posible. Se generan advertencias sobre el riesgo que representan dichas violencias para el orden moral, las instituciones, los poderes sociales y políticos. Como advierte Eyerman (2011), se construyen narraciones donde los supuestos perpetradores y sus víctimas son nombrados y asimétricamente posicionados. A partir de estas narrativas sobre la violencia, la sociedad cuestiona su identidad e incluso a veces los soportes que permiten la solidaridad y la cohesión social. La violencia tensiona, como siguiere Smelser (2004), las instituciones en su conjunto y marca la memoria colectiva.

\section{La denuncia y el suicidio}

Una de las primeras expresiones del \#Metoo en México se manifestó a través del \#Yaestuvo en 2017, organizado por mujeres dedicadas a la comedia y que al final de ese año reportó más de 100 denuncias. ${ }^{10}$ Ese mismo año surgieron otras denuncias en redes sociales bajo etiquetas como: \#NoEsNormal, \#NiUnaMas \#NiUnaMenos, \#MeToo, o \#YoTambién. ${ }^{11}$ En 2018 la periodista Carmen Aristegui presentó varios casos de víctimas de acoso. ${ }^{12}$ Las denuncias

10. Agencia AP (28 de noviembre de 2017): "Mujeres comediantes en México alertan sobre violencia de género en su industria" (EI Comercio). Recuperado de https://www.elcomercio.com/tendencias/mujeres-comediantes-mexicoviolenciadegenero-industria.html

II. Fundar Centro de Análisis (2017). Contra la violencia que no se ve. Recuperado de https://fundar.org.mx/contra-la-violencia-que-no-se-ve/

12. Redacción (2018). Los rostros del \#MeToo en México: Del acoso a la indignación. Aristegui Noticias. Recuperado de https://aristeguinoticias.com/0803/ mexico/los-rostros-del-metoo-en-mexico-del-acoso-a-la-indignacion/ 
fueron puestas en duda y las mujeres que ofrecieron sus testimonios sufrieron agresiones en redes sociales. Esto dio lugar al movimiento \#Yonodenuncioporque, con el que se evidenciaban los obstáculos para denunciar el acoso contra las mujeres.

Un año después, el 21 de marzo de 2019, Anna G. González acusó al escritor Herson Barona de golpear a varias mujeres y pidió a otras víctimas del escritor hacer una denuncia pública. ${ }^{13} \mathrm{El}$ acusado negó los señalamientos que hicieron en su contra. La denuncia de González fue replicada utilizando el \#Metoo para señalar casos de acoso en el círculo del arte, la ciencia, la comunicación y la academia. El 22 de marzo surgió el \#Metooescritoremexicanos junto con la cuenta@metooescritores, con el que se denunciaba a presuntos acosadores del gremio literario. Desde esta cuenta se crearon distintas etiquetas para denunciar el acoso, la violencia sexual, así como para exigir derechos laborales de las mujeres (Esquivel, 2019). Poco a poco se sumaron más cuentas, entre otras: @MeTooCineMx, @ MeTooPeriodista, @MeTooCreativos, @metoomusicamx, @ MeTooFotografos, @metooartesmx, @MeTooEmpresario, @metootechmx, @MeTooAbogadosMx, @MeTooMedicina, @MeTooActivista1, @metootuiteros, @metooteatromx, @ MeTooJalisco, @MeTooAcademicos,@MeTooPoliticos, @ MeTooAgenciasMx y@MeTooCulturaMex. De igual forma, en universidades públicas y privadas del país se retomaron los "tendederos contra el acoso" (exposición de nombres de presuntos acosadores con la descripción de las situaciones de agresión en lugares visibles en los campus).

Los medios destacaron que las denuncias a través del \#Metoo mexicano en 2018 se circunscribieron a la industria del entretenimiento (Nicolás et al., 2019). Sin embargo,

13. Ávila,Y.(2019). ¿Cómo surgió el movimiento MeToo y cómo revivió en México? Animal Político. Recuperado de https://www.animalpolitico.com/elsabueso/comosurgio-el-movimiento-me-too-y-como-revivio-en-mexico/ 
para 2019 se amplió a otros espacios como la música, la academia y otros sectores considerados - a decir de este posicionamiento - como ajenos a las dinámicas de acoso por una aparente posición más abierta y liberal respecto a los temas de sexualidad (Peñaloza, 2019a; Maza, 2019; Fuentes, 2019; Zamarrón, 2019; Vela, 2019). Conforme el movimiento tomó fuerza, surgieron voces a favor y en contra.

Quienes estaban a favor del \#Metoo, enfatizaron la necesidad de continuar con las denuncias para impulsar una revolución feminista (Maza, 2019); se invitó a otras mujeres a romper el silencio, señalando a los acosadores y llevándolos a la justicia (Vela, 2019; Peñaloza, 2019a). Algunos articulistas interpretaban que todas las denuncias deberían ser consideradas como ciertas, sin dudar de los hechos que narraban las víctimas (Peñaloza, 2019a). En la medida en que haya más denuncias — sostuvieron algunos periodistas - se podrá comprender cómo se relaciona nuestra sociedad con el sexo, el deseo y la frustración; insumos necesarios para empujar una transformación social (Zamarrón, 2019a; Fuentes, 2019). Asimismo, quienes estaban a favor consideraron que las denuncias del \#Metoo habían permitido mostrar la incapacidad de las instituciones de justicia para atender a las víctimas de acoso o violencia sexual (Vela, 2019; Fuentes, 2019).

Los posicionamientos en contra advirtieron que había testimonios que señalaron directamente a su acosador, dejando atrás el anonimato. Desde esta postura se consideró que algunas acusaciones parecían estar motivadas por el despecho y enojo con la ex pareja o por difamación (Guarneros, 2019). De esta forma se interpretó que la ola de acusaciones por acoso colocó en los medios al \#Metoo, debido a la denuncia de personas que se sabía acosaban, y de nuevos presuntos acosadores o abusadores (Peñaloza, 2019a). Estos comentaristas sugerían que las denuncias en algunos espacios laborales, como los educativos, salían del 
Twitter para cristalizarse en tendederos y procedimientos normativos de carácter laboral, en la forma de una nueva aplicación de justicia.

Las denuncias en las redes revelaron agresiones físicas, miradas consideradas como lascivas, tocamientos inadecuados, intimidaciones, abusos y presiones para obtener favores sexuales a cambio de ganar una plaza, ascender en el trabajo, mejorar una calificación o sus ingresos $u$ obtener un papel en el cine. Algunos acusados aceptaron su responsabilidad y ofrecieron disculpas, otros no. Hubo acusados que fueron sancionados o amonestados, otros fueron despedidos, removidos u obligados a renunciar a su empleo (Rivera, 2020). La mayor parte de los acusados perdieron su prestigio y autoridad. Frente a esta ola de denuncias, en algunas columnas se opinó que era necesario analizar su contexto y las sanciones:

[...] salvo que sea punible judicialmente, lo prudente será tomar esto [las denuncias] más como un ejercicio de focos rojos. No como un linchamiento, sino como una mirada al futuro: tener cuidado con ciertas personas, esperar que esto desaliente a los abusadores o los haga cambiar (Peñaloza, 2019a).

El 31 de marzo se acusó — a través de @metoomusicamx-a Armando Vega-Gil, integrante del grupo de rock Botellita de Jerez y escritor de libros y cuentos para niños. ${ }^{14}$ Una mujer de 27 años lo acusó de haberla invitado a su hogar 14 años antes, cuando ella tenía 13 años, y de haberle hecho sentir incómoda con una serie de insinuaciones de carácter sexual.

A decir de la presunta víctima, Vega-Gil se aprovechó de su fama como músico para obtener su número telefónico

14. La cuenta @metoomusicamx se presentaba con la siguiente frase: "Espacio abierto para denunciar agresiones en la industria de la música. Manda un DM con tu denuncia anónima y publicamos el nombre del agresor". MetooMúsicosMexicanos. (I de abril de 2019). Publicado en Twitter. Recuperado de https://Twitter.com/ mariomonroymmty/status/ I | 282 I09 |3 | 4094080/photo/ I 
cuando ella se acercó para pedirle un autógrafo y una fotografía en un concierto. "Se aprovechó de mi ingenuidad y me engatusó [...] me decía que yo era diferente a las de mi edad, que era muy especial". ${ }^{15}$ La denunciante señaló que el músico la invitó a su casa y ella fue con una amiga, allí les tomó fotografías que les hicieron sentirse muy incómodas porque "nos pedía que nos acomodáramos y todo". ${ }^{16}$ Ella compartió su sensación de incomodidad y los supuestos mensajes que Vega-Gil le enviaba acosándola: "Me decía cosas cada vez más asquerosas y explícitamente sexuales, hasta que me dio muchísimo miedo; decidí bloquearlo y cambiar de teléfono". ${ }^{17}$ La víctima advirtió:

Si hubiera tenido un gramo más de inocencia y hubiera ido a su casa sola, estoy segura que ese viejo hubiera abusado de mi. [...] Estoy segura que no soy la única [...] El señor se dedica a escribir libros para niños y adolescentes $y$ son historias que si conoces el lado perverso del autor, resultan muy siniestras. ${ }^{18}$

Vega-Gil rechazó las acusaciones. Señaló que era una persona pública y que constantemente recibía muchas menores de edad en su casa [...] "y mantengo comunicación con ellas y ellos”. ${ }^{19}$ El músico expresó: "escribo canciones para menores y soy padre de familia que apoya activamente la defensa de los derechos universales de la infancia”. Reconociendo el carácter legítimo de la denuncia anónima, Vega-Gil señaló que había invitado a la víctima a que hablaran del asunto,

I5. PuentesGlz. (I de abril de 2019). Publicado en Twitter. Recuperado de https:// Twitter.com/PuentesGlz/status/ I I 2765475585474562/photo/ I

16. PuentesGlz. (I de abril de 2019). Publicado en Twitter. Recuperado de https:// Twitter.com/PuentesGlz/status/ I I 2765475585474562/photo/ I

17. PuentesGlz. (I de abril de 2019). Publicado en Twitter. Recuperado de https:// Twitter.com/PuentesGlz/status/ I I 2765475585474562/photo/ I

I8. PuentesGlz. (I de abril de 2019). Publicado en Twitter. Recuperado de https:// Twitter.com/PuentesGlz/status/ I I 2765475585474562/photo/ I

19. ArmandoVegaGil. (I de abril de 2019). Publicado en Twitter. Recuperado de https://Twitter.com/ArmandoVegaGil/status/ I | 266622295 | 39|233/photo/ I 
ella con pruebas y testigos, y acompañada por asesores y las chicas del \#MetooMúsicosMexicanos, para que estuviera segura de que no habría amenazas y represalias. ${ }^{20} \mathrm{El}$ músico defendió al movimiento, así como el derecho a las mujeres a denunciar a sus agresores. Pero advirtió que con la denuncia en su contra, todo estaba perdido para él: su trabajo y su imagen pública. Así que anunció por Twitter que se suicidaría, advirtiendo que no se culpara a nadie de su decisión (Rodríguez et al., 2019). Ofreció finalmente una disculpa a las mujeres si en algún momento las ofendió con su modo machista, y aseguró: "los machos, somos criaturas de nuestros tiempos". ${ }^{21}$ El cuerpo sin vida de Vega-Gil apareció colgado pocas horas después en un árbol cerca de su domicilio.

Una vez que la muerte del músico se hizo pública, la cuenta de \#MetooMúsicosMexicanos informó que había hablado con Vega-Gil para platicar, pero que no tuvieron respuesta, sino una carta de suicidio, por lo que - aseguraron- él sabía que era culpable: "Fue chantaje mediático". ${ }^{22}$ Acusaron que la muerte del músico era responsabilidad de él y de nadie más. La cuenta desapareció algunas horas después, sus administradoras denunciaron que había sido hackeada para sacarlas de la red, y advirtieron: "No nos vamos a callar. Ahora más que nunca, seguimos en apoyo a las víctimas y también a las que aún no se atreven a alzar la voz". ${ }^{23}$ Aseguraron que fueron dañadas y agredidas por los medios de comunicación "que intenta[ron] hacernos sentir como si la muerte de Armando Vega-Gil fuera nuestra culpa.

20. ArmandoVegaGil. (I de abril de 2019). Publicado en Twitter. Recuperado de https://Twitter.com/ArmandoVegaGil/status/ I I I 266622295 I 39 I 233/photo/ I 2I. ArmandoVegaGil. (I de abril de 2019). Publicado en Twitter. Recuperado de https://Twitter .com/ArmandoVegaGil/status/ I I 266622295 I 39 I233/photo/ I 22. Editor. (I de abril de 2019). Milenio Digital. Recuperado de https://www.milenio. com/cultura/too-musicos-mexicanos-vuelve-Twitter -acusa-censura

23. Redacción. (2 de abril de 2019) BBC News Mundo. Recuperado de https://www. bbc.com/mundo/noticias-america-latina-47786292 
Nosotras no lo matamos. Fue un suicidio. Sepan que esto es también violencia y no lo notan porque está normalizada". ${ }^{24}$ Posteriormente volvió a aparecer la cuenta y cerró definitivamente el 3 de abril. En su última publicación se leyó:

Lamentamos profundamente el suicidio de ArmandoVega Gil. Nuestra reacción inicial no fue la adecuada y, por ello, extendemos una disculpa honesta a todos sus allegados [...] Jamás incitamos a alguien a hacerse ningún daño ni a acabar con su vida en lugar de afrontar los hechos ante las autoridades. ${ }^{25}$

Este acontecimiento generó una disputa en los medios de comunicación sobre las consecuencias de las acusaciones anónimas a través de \#Metoo. Hubo quienes responsabilizaron al movimiento de empujar a Vega-Gil al suicidio, mientras que otros arguyeron que el acto evidenciaba su culpabilidad. Algunos argumentaron que cualquier expresión del \#Metoo terminaba por convertirse en un mecanismo de chantaje, acoso y venganza contra figuras públicas, contaminando así su pretensión de llevar justicia a las víctimas de acoso. Otros señalaron que \#Metoo debía mejorar su operación debido a las probabilidades de que hubiera más acusaciones. De esta forma, se estructuró una disputa en la esfera civil en la que se defendió y criticó un mecanismo que buscaba operar como un instrumento para reparar el daño ocasionado a las mujeres víctimas de acoso.

\section{La disputa y las atribuciones de sentido}

Después del suicidio de Vega-Gil se desplegaron tres discursos en competencia para significar la muerte del artista, así como la pureza e impureza del \#Metoo. El

24. Redacción. (2 de abril de 2019). BBC News Mundo. Recuperado de https://www. bbc.com/mundo/noticias-america-latina-47786292

25. Editor. (3 de abril de 2019). \#MeTooMúsicosMexico suspende su actividad en Twitter. EDT. Recuperado de https://estadodeltiempo.mx/metoomusicosmexicosdesaparece-de-Twitter/ 
primero, sugirió que el suicidio era para desprestigiar al movimiento. Por tanto, \#Metoo no debía cejar en su apoyo a las víctimas del machismo (Albarrán, 2019; Lamas, 2019; Alanís, 2019b; Editorial El Universal, 2019; Rojas, 2019; Sefchobich, 2019; Tello, 2019; Buendía, 2019; Zuckermann, 2019; Editorial La Jornada, 2019). Desde esta visión era importante apoyar al movimiento sin condiciones, ya que permitió a las mujeres denunciar de forma anónima a sus agresores. Se afirmó que estos últimos tendrían que defenderse y si fueran encontrados culpables, tendrán que pagar (Becerra, 2019; Loaeza, 2019; Alanís, 2019b; Guarneros, 2019). Se admitió que quizás algunas mujeres - muy pocas, se enfatizó- podrían difamar a ciertos hombres, pero se consideró que eso era un mal menor si con eso se acaba con el machismo (Becerra, 2019). El \#Metoo logró — a decir de esta interpretación - que los hombres se la piensen mejor antes de acosar o violentar a una mujer (Rojas, 2019; Guarneros, 2019). Con la muerte de Vega-Gil — se advirtió— se quiere desprestigiar un movimiento auténtico de miles de mujeres que han sufrido acoso, desviando la atención sobre el supuesto carácter perverso de la denuncia anónima, cuando esta última es el recurso que queda a las mujeres cuando quieren hacerse escuchar. Desde esta postura se consideró que había que decirle al país que \#Metoo no tuvo la culpa de la muerte del músico (Sierra, 2019b).

Un segundo discurso valoró que \#Metoo era un movimiento legítimo que desafortunadamente se contaminó por activistas fanáticas que buscan polarizar a la sociedad (Revueltas, 2019). Según este discurso, las denuncias en Twitter fueron cooptadas por actores políticos que creen que una parte del país está formada por personas que encarnan la bondad y, por otra parte, por sujetos que encarnan el mal. Los primeros son considerados perfectos y puros, mientras que los segundos son eterna malignidad (Revueltas, 2019; Malvido, 2019). Según este discurso, este tipo de posturas 
refleja un fanatismo que convierte movimientos liberadores en opresores, como sucedió con \#Metoo, éstos no buscan justicia, sino el castigo de cualquier persona sospechosa de haber hecho algo en el pasado, incluso si los hechos ocurrieron hace 30 años (Revueltas, 2019; Clariond, 2019). En este sentido, se interpretó que el discurso centrado en la venganza y el odio de algunas feministas del \#Metoo prefigura el futuro de una sociedad asfixiante, sometida al rencor y la sospecha permanente (Revueltas, 2019: 5; Lavín, 2019; Peñaloza, 2019b). Desde esta perspectiva se opinó que vendría un futuro carente de justicia si se considera que quienes denunciaron desde el anonimato celebraron el suicidio de Vega-Gil (Musacchio, 2019; Pretrich, 2019; Villeda, 2019).

Un tercer discurso responsabilizó directamente al\#Metoo de provocar el suicidio del músico. Se afirmó que el espíritu y la estrategia que da forma a este movimiento tienen como objetivo acusar y juzgar sin pruebas suficientes. Se dijo que \#Metoo ponía a los hombres frente a una situación en la que no pueden opinar ni defenderse. Desde esta posición se consideró que aquello era comprensible si se considera el daño que han provocado a las mujeres. Pero eso ha derivado - se alertó- en una actitud pasiva que en ningún momento pone en duda la credibilidad de las mujeres cuando acusan a los hombres - tengan o no pruebas-. Estos últimos tienen que soportar la lapidación y el linchamiento, tienen prohibido defenderse y, por supuesto, nadie los defenderá porque pueden acabar en el banquillo de los acusados, se dijo (Clariond, 2019). Los columnistas argumentaron que los efectos de una denuncia de este tipo son devastadores, porque cancelan el derecho de abogar por la inocencia, teniendo que renunciar a la vida pública y al trabajo. Desde esta interpretación, las acusaciones anónimas visibilizan el acoso, pero pueden lastimar la reputación de personas inocentes (García, 2019; Editorial La Jornada, 2019), como 
sucedió a Vega-Gil (Gamés, 2019; Sarmiento, 2019; Editorial La Jornada, 2019).

Para este posicionamiento en contra del \#Metoo, el suicidio del bajista de Botellita de Jerez mostró que la lucha contra el acoso se transformó en acoso: dejó ver que las acusaciones - verdaderas o falsas- pueden terminar con la vida de una persona que decide sacrificarse en su desesperación por no poder defenderse (Lavín, 2019; Peñaloza, 2019b).

El argumento de esta postura es que las acusaciones sin pruebas terminan en linchamientos, juicios sumarios y consignas moralizantes sobre el comportamiento sexual de hombres y mujeres (Gamés, 2019; Zamarrón, 2019a, 2019b; Buendía, 2019; Peñaloza, 2019b). Algunos columnistas tomaron como referencia al movimiento francés La Otra Palabra, para afirmar que no son un delito ni la galantería ni la seducción insistente o torpe (Gamés, 2019; Musacchio, 2019; Editorial La Jornada, 2019). Se afirmó que el suicidio de Vega-Gil acabó por colocar tantas vidas a la intemperie - como a las víctimas del acoso-y lastimar a muchas más — como a la familia del músico— (Tello, 2019; Buendía, 2019; Malvido, 2019). Desde esta posición se consideró que era necesario distanciarse de las visiones dicotómicas que ponen a las mujeres como víctimas y a los hombres como victimarios (Malvido, 2019).

Se advirtió que el suicidio de Vega-Gil no puede pasarse por un mero accidente atribuido a su condición emocional. A decir de este discurso contra \#Metoo, hay un riesgo real de que las denuncias anónimas terminen por empujar a alguien a quitarse la vida sin que las denunciantes asuman algún grado de responsabilidad —incluso si el músico enfatizó que\#Metoo no tenía la culpa de su decisión-(Rocha, 2019; Peñaloza, 2019b). Desde esta posición se asumió que\#Metoo no frenó sus acusaciones cuando Vega-Gil escribió en las redes que iba a suicidarse. Desde esta opinión, aquél era el

\section{6}


momento para que \#Metoo llamara a la reflexión y dijera que nadie acuse a nadie, víctimas hay en todos los bandos, alto (Pretrich, 2019). La reacción del \#Metoo fue calificada de cobarde y ruin al afirmar que el músico quería jugar con su suicidio para salvarse de una demanda por pederastia y limpiar su imagen. A decir de ciertos comentaristas, \#Metoo mexicano decidió hundirse en sus propios errores (Pretrich, 2019; Villeda, 2019).

Otros columnistas opinaron que el movimiento había visibilizado una serie de tensiones en la sociedad mexicana. Las denuncias y la muerte del músico mostraron la doble personalidad del mundo de las artes y la academia — con un discurso progresista que oculta su misoginia-. También se opinó que el movimiento sacó a la luz la doble personalidad de las feministas: una que exige justicia y reparación del daño, otra que se mueve por el odio (Villeda, 2019). En el mismo orden de ideas, se valoró que reflejaba el fracaso del Estado de derecho, debido a que la ineficiencia del Estado empujaba a las mujeres a denunciar de forma anónima, y era incapaz de garantizar la presunción de inocencia de los acusados (Alanís, 2019a; Villanueva, 2019; Editorial La Jornada, 2019; Zuckermann, 2019; Peñaloza, 2019b; Poniatowska, 2019). Para otros articulistas, \#Metoo evidenció el alto grado de conservadurismo, machismo y prácticas patriarcales que pueblan las relaciones entre hombres y mujeres en México (Zamarrón, 2019b; Clariond, 2019; Lamas, 2019; Editorial El Universal, 2019; Sierra, 2019a).

Algunas interpretaciones concluían que \#Metoo sólo podría sobrevivir si continuaba visibilizando el acoso, pero asumiendo que la justicia sólo es posible sin venganza (Villera, 2019). Asimismo, se afirmó que la muerte de Vega-Gil debía servir para mejorar el \#Metoo y no para silenciarlo (Monreal, 2019; Sierra, 2019a; Virrueta, 2019; Peñaloza, 2019b). Hubo un acuerdo en varias columnas sobre la necesidad de atender las causas del acoso para que 
el movimiento no se transforme en la inquisición para los hombres ni en un medio para victimizar una y otra vez a las mujeres - cancelando su derecho a la denuncia anónima(Monreal, 2019; Sierra, 2019a; Virrueta, 2019; Peñaloza, 2019b). También se consideró que era necesario construir una amplia y robusta agenda de equidad de género que involucre a los hombres (Clariond, 2019; Sierra, 2019a). Una agenda que permita distinguir lo que es un acoso de lo que no es: ¿una mirada, unas palabras, son acoso? (Lamas, 2019). Desde este posicionamiento se enfatizó que si no se diferencia claramente este punto se podría dar paso a una narrativa puritana peligrosa para las libertades en todos los sentidos y no sólo en el ámbito de las relaciones entre ambos sexos (Lamas, 2019; Lavín, 2019).

Varios articulistas concordaron en que el acoso es un problema grave en el país, por lo que eran de esperarse otras olas de \#Metoo, por lo cual llamaban a mejorar la educación digital en redes — particularmente en Twitter - para evitar la tergiversación de comentarios que terminan por polarizar el debate público (Guarneros, 2019). Según esta opinión, la muerte de Vega-Gil debía ser una oportunidad para mejorar la forma en cómo se transmiten los mensajes a través de las redes sociales (Cueva, 2019; Zamarrón, 2019b; Editorial La Jornada, 2019). Se sugirió que el movimiento debía seguir denunciando el acoso sin generar campañas de odio que criminalicen injustamente a los presuntos victimarios (Gamés, 2019). Desde esta postura se sugirió la implementación de protocolos que eviten falsas denuncias (Rojas, 2019; Sierra, 2019a). Se recomendó implementar un dispositivo institucional controlado por el Estado para garantizar, al mismo tiempo, el anonimato de la víctima y la presunción de inocencia del acusado (Lavín, 2019; Rojas, 2019; Buendía, 2019; Virrueta, 2019) — por ejemplo, a través de investigar de oficio las denuncias de acoso y determinar la responsabilidad o no del acusado (Tello, 2019). 


\section{Los binarios en competencia}

La disputa simbólica por significar el \#Metoo y la muerte de Vega-Gil se articuló a partir de un sistema binario de sentido. Quienes apoyaron la denuncia a través del \#Metoo consideraron que su contribución más relevante fue dar voz a las sin voz, garantizando el anonimato y evitando la revictimización. Esta posición valoró como positivo el testimonio de las víctimas por encima de las evidencias. En este sentido, para esta postura es más importante la sanción social hacia los presuntos acosadores que la justicia en tribunales. Para este posicionamiento el desprestigio de los acusados y de las instituciones — que aparentemente los protegen- es la vía para la reparación civil del daño que han sufrido las mujeres. El desprestigio opera como una forma de justicia horizontal que pone en evidencia la verticalidad, normatividad y funcionamiento heteropatriarcal del sistema de justicia. De esta forma, \#Metoo fue considerado como una movilización civil, caracterizada por su racionalidad, realismo, autonomía, honestidad, capaz de generar vínculos abiertos, críticos y francos, que promueve la ampliación de lazos de solidaridad e inclusión ciudadana. Que apeló a una reparación del daño producido por normas sociales anticiviles — no racionales, deferentes y opacasque normalizan el machismo, las insinuaciones sexuales y la coerción.

Quienes criticaron al movimiento - aunque justificaron sus demandas - advirtieron que se había contaminado por actores anticiviles. Señalaron que la tergiversación del espíritu civil del movimiento se produjo cuando comenzaron a valorar positivamente la venganza y la sanción social y moral por encima de la aplicación de la ley y la reparación judicial del daño. Desde este posicionamiento crítico, las mujeres detrás del \#Metoo estaban motivadas por sus sentimientos o pasiones y la pretensión de modificar a fondo 
las estructuras y las instituciones de justicia. Este posicionamiento valoró como contraproducente el desdén del movimiento por el marco legal, ya que negó la presunción de inocencia y el debido proceso de los acusados, propiciando su linchamiento mediático. Los críticos del \#Metoo acusaron que transitó de la pretensión de reparación civil, al acoso digital contra los presuntos acosadores.

Finalmente, un tercer posicionamiento atribuyó al \#Metoo un origen anticivil y criticó que las denuncias a través de Twitter asumieran la presunción de culpabilidad por encima de la presunción de inocencia. Para esta postura, si bien el sistema de justicia y su funcionamiento revictimiza a las mujeres, no se puede acusar a nadie sólo con testimonios, sin aportar pruebas o sin la versión del acusado. Esta postura cuestionó el hecho de que \#Metoo pusiera por delante la sanción social frente a la legal, cuando había que garantizar los mecanismos judiciales que protejan los derechos de las víctimas y de los acusados. Según esta línea discursiva, estos procedimientos regulativos de orden legal son los únicos que podrían permitir la reparación del daño a las víctimas de acoso. La turba mediática que dispararon las denuncias de acoso no contribuye — según esta interpretación - a la consolidación de una sociedad basada en leyes e instituciones.

En la figura 1 se pueden apreciar las estructuras binarias de los discursos en competencia que marcaron la disputa por el \#Metoo y la muerte de Vega-Gil. Destaca la confrontación respecto al carácter civil o anticivil — sagrado o contaminante - de: la denuncia anónima o abierta; del testimonio frente a la prueba; de la sanción social contra lo legal; la venganza frente a la justicia; de la pasión frente a la razón; de la culpabilidad o la inocencia. Estas relaciones binarias permiten apreciar tres estructuras narrativas del debate y ayudan a comprender el campo de oportunidad y las difi- 
cultades que existen para establecer discursos y narrativas que permitan entrelazar las posiciones en competencia.

Figura 1

Discurso binario sobre el \#Metoo (México, 2019)

\begin{tabular}{|c|c|c|c|c|c|c|c|c|c|c|c|}
\hline & \multicolumn{4}{|c|}{$\begin{array}{c}\text { En apoyo a } \\
\text { \#Metoo }\end{array}$} & \multicolumn{3}{|c|}{$\begin{array}{c}\text { Posición crítica } \\
\text { \#Metoo }\end{array}$} & \multicolumn{4}{|c|}{$\begin{array}{c}\text { En contra de } \\
\text { \#Metoo }\end{array}$} \\
\hline+ & Voz & Anonimato & Testimonio & $\begin{array}{c}\text { Sanción } \\
\text { Social }\end{array}$ & Justicia & Reparación & $\begin{array}{l}\text { Razón/ } \\
\text { Mesura }\end{array}$ & $\begin{array}{l}\text { Presunción de } \\
\text { Inocencia }\end{array}$ & Pruebas & $\begin{array}{l}\text { Sanción } \\
\text { Judicial }\end{array}$ & Instituciones \\
\hline - & Silencio & Victimización & Pruebas & $\begin{array}{l}\text { Sanción } \\
\text { Judicial }\end{array}$ & Castigo & Venganza & $\begin{array}{c}\text { Pasión/ } \\
\text { Odio }\end{array}$ & $\begin{array}{l}\text { Presunción de } \\
\text { Culpabilidad }\end{array}$ & Testimonio & $\begin{array}{c}\text { Sanción } \\
\text { Social }\end{array}$ & Linchamiento \\
\hline
\end{tabular}

Fuente: elaboración propia.

Este análisis obliga a pensar cómo continuar un debate social donde, por un lado, se ve como positivo el testimonio por encima de la prueba y, por otro, se considera que quienes hacen las denuncias las mueve la venganza y las emociones y no la pretensión de justicia, reparación y mesura. Más aún, cómo se defiende la presunción de inocencia, las pruebas y las instituciones cuando el sistema de justicia revictimiza a las mujeres a tal punto que opera contra la reparación del daño frente el acoso. Cada una de estas posiciones llama, a su manera, a formas amplias de solidaridad e inclusión social. No obstante, cada una atribuye a sus contrapartes la supuesta defensa de posicionamientos de solidaridad e inclusión particularistas y excluyentes.

\section{Conclusión}

En este artículo se analizó la disputa de sentido en torno a las denuncias de acoso contra las mujeres por el \#Metoo en México en 2019, particularmente a la luz del suicidio de uno de los presuntos acosadores. Se examinó cómo en las columnas periodísticas se apoyaron, criticaron y denostaron las denuncias por acoso realizadas a través de Twitter, prestando atención a cómo dichas columnas atribuían motivos, 
relaciones y adscripciones institucionales civiles o anticiviles al movimiento \#Metoo. Los discursos compitieron por establecer la legitimidad y pureza del movimiento a partir de la demanda de reparación del daño hacia las mujeres, la violación a la presunción de inocencia de los presuntos acosadores, así como la necesidad de pensar formas alternativas de denuncia. Cada uno de los posicionamientos respecto al\#Metoo plantearon y exigieron distintos modelos de solidaridad ciudadana y justicia frente al acoso a las mujeres; es decir, apelaron a diferentes formas de reparación civil y ejercicio de la justicia.

Cuando Vega-Gil decidió quitarse la vida por las acusaciones de las que fue objeto, las atribuciones de pureza e impureza asignadas desde diferentes discursos al \#Metoo se acentuaron significativamente. Así, el \#Metoo fue sometido a escrutinio en la prensa por posiciones, al parecer, irreconciliables. El señalamiento de que la denuncia por Twitter era legítima e incluso necesaria ante la falta de mecanismos institucionales para proteger a las mujeres, se confrontó con los señalamientos que advirtieron que \#Metoo sólo buscaba el linchamiento digital de los supuestos agresores sin ningún tipo de prueba, lo cual también podría ser considerado - desde esta perspectiva - como una forma de violencia. En medio de estas posiciones encontradas hubo voces que consideraron que más bien los principios que habían propiciado la emergencia de \#Metoo habían sido contaminados por el rencor y la venganza de un grupo particular de mujeres, por lo cual era necesario replantear el movimiento y buscar alternativas sociales e institucionales para enfrentar el acoso.

Esta disputa permite comprender el enorme desafío que tiene que enfrentar la justicia feminista ante casos de violencia. Si bien las tres posiciones no cuestionan la justa demanda de las mujeres de castigar el acoso, hay desacuerdos sobre mecanismos como el \#Metoo para enfrentarlo.

\section{2}


Como se ha visto en este texto, los desacuerdos giran en torno al carácter civil o anticivil de los medios utilizados, generando una disputa en la esfera civil que tiene efectos en las dinámicas estructurales de la inclusión y la exclusión social, así como los retos hacia futuro para garantizar una sociedad más justa, equitativa y libre de violencia para las mujeres.

La reconstrucción de las narrativas y discursos sobre este tipo de disputas resulta importante en la medida en que en los últimos años se han podido observar dos grandes movilizaciones colectivas en México — aparte de la que aquí se ha analizado - en contra de la violencia, el acoso y la dominación de las estructuras heteropatriarcales. Una de ellas tuvo lugar en el mes de agosto de 2019 — apenas cinco meses después de la ola de denuncias del \#Metoo- bajo la consigna "No me cuidan, me violan", y en marzo de 2020 se llevó a cabo el Paro Nacional de Mujeres. El conjunto de estas movilizaciones ha desatado una y otra vez disputas en la esfera civil (Cardona y Arteaga, 2020), movilizando discursos y narrativas en competencia que tratan de establecer el carácter legítimo o ilegítimo de las diferentes expresiones de las protestas feministas.

Acker, J. (2006). Inequality regimes: Gender, class, and race Bibliografía in organizations. Gender and Society, 20(4): 44I-464. doi: |0.1 |77/089|243206289499

Alanís, E. (2019a, 5 de abril). AMLO se impuso, "haiga sido como haiga sido". El Universal. Recuperado de https:// www.eluniversal.com.mx/columna/elisa-alanis/nacion/ amlo-se-impuso-haiga-sido-como-haiga-sido

—. (2019b, 12 de abril).\#MeToo y el acceso a la justicia. El Universal. Recuperado de https://www.eluniversal.com. $\mathrm{mx} /$ articulo/maria-del-carmen-alanis/nacion/metoo-yel-acceso-la-justicia 
Bibliografía

Albarrán, J. (2019, 5 de abril). Lo seguro ya no tiene misterio. Milenio. Recuperado de https://www.milenio.com/ opinion/jairo-calixto-albarran/politica-cero/lo-seguro-ya-no-tiene-misterio

Alexander, J. (2006). The Civil Sphere. Oxford: Oxford University Press.

- (2008). Civil sphere, state, and citizenship: Replying to Turner and the fear of enclavement. Citizenship Studies, I2(2): I85-I94. doi: I0.1080/ | 362 I02080 I900| 43

_. (20I5). Nine theses on the Civil Sphere. En P. Kivisto y G. Sciortino (eds.), Solidarity, Justice and Incorporation (pp. 172-190). Oxford: Oxford University Press.

—. (2017). The Drama of Social Life. Oxford: Oxford University Press.

- (20I8). The Societalization of Social Problems: Church Pedophilia, Phone Hacking, and the Financial Crisis. American Sociological Review, 83(6): 1049-1078. doi: I0.1I77/0003 I224I8803376

Alexander,..,y Mast,J.(20I I).The cultural pragmatics of symbolic action. En J.Alexander (ed.), Performance and Power (pp. 7-24). Cambridge: Cambridge University Press.

Alexander, J., y Tognato, C. (2018). Introduction: For Democracy in Latin America. En J. Alexander y C. Tognato (eds.), The Civil Sphere in Latin America (Pp. I-16). Cambridge: Cambridge University Press.

Arteaga, N., y Cardona, L. (2020). La disputa por el acoso en la esfera civil:\#Metoo y la Une autre parole. Nóesis. Revista de Ciencias Sociales y Humanidades, 29(58): I-23. doi: http://dx.doi.org/ 10.20983/noesis.2020.3.I

Arzuaga, J., y Arteaga, N. (2017). Liturgia republicana y contra-performances: Protestas y pugnas en la investidura presidencial de 2012 en México. Revista Mexicana de Ciencias Políticas y Sociales, nueva época, I (229): I 49- I80. Universidad Nacional Autónoma de México.

Augé, M. (2010). Carnet de routes et de déroutes. París: Galilée. 
Basu, K. (2003).The economics and law of sexual harassment Bibliografía in the workplace. Journal of Economic Perspectives, I 7(3): I4 I-I57. doi: I0. I257/089533003769204399

Becerra,J. (2019,8 de abril).\#MeToo en México y yo como mujer... Milenio. Recuperado de https://www.milenio. com/opinion/juan-pablo-becerra-acosta/doble-fondo/ metoo-en-mexico-y-yo-como-mujer

Bowman, J. (2020). Maximizing \#MeToo: Intersectionality and the Movement. Georgetown Law Faculty Publications and Other Works, núm. 228I. Recuperado de https:// scholarship.law.georgetown.edu/facpub/228I

Buendía, J. (2019, 7 de abril). \#MeToo México ¿continuará? Excélsior. Recuperado de https://www.excelsior.com.mx/ opinion/jose-buendia-hegewisch/metoo-mexico-continuara/I 306247

Butler, E., y Luengo, M. (2016). Conclusion: News innovation and enduring commitments. En J.Alexander, E. Butler y M. Luengo (eds.), The Crisis of Journalism Reconsidered (pp. 282-290). Cambridge: Cambridge University Press.

Cadei, E. (2017,25 de octubre). Few in Washington are saying \#MeToo. California congresswoman wants to change that. Miami Herald. Recuperado de https://web.archive. org/web/20 I 7 I 02605433 I/http:/www.miamiherald. com/news/politics-government/article I 807679 I I.html

Cardona, L., y Arteaga, N. (2020). “No me cuidan, me violan": La esfera civil y la protesta feminista. Región y Sociedad, núm. 32, p. el345. https://doi.org//0.22 I98/ rys2020/32/1345

Cedeño, A. (2019, 2 de abril). \#MeToo o cómo detener el incendio de Roma en la era de la posverdad. El Universal. Recuperado de https://www.eluniversal.com.mx/ columna/alonso-cedeno/nacion/metoo-o-como-detener-el-incendio-de-roma-en-la-era-de-la-posverdad 
Bibliografía

Clariond,A. (2019, 3 de abril). Prohibido quejarse. Reforma. Recuperado de https://www.reforma.com/prohibido-quejarse-2019-04-03/opI53899

Clark, R. (2019). "I See You, I Believe You, I Stand with You": \#MeToo and the performance of networked feminist visibility. Feminist Media Studies. doi: I0.1080/ I 4680777.2019.1628797

Cueva, Á. (2019, 7 de abril). La muerte y \#MeToo. Milenio. Recuperado de https://www.milenio.com/opinion/alvaro-cueva/ojo-por-ojo/la-muerte-y-metoo

D'Zurilla, C. (2017, 16 de octubre). In saying \#MeToo, Alyssa Milano pushes awareness campaign about sexual assault and harassment. Los Angeles Times, Recuperado de https://web.archive.org/web/20I7I0I707564I/ http:/www.latimes.com/entertainment/la-et-entertainment-news-updates-metoo-campaign-me-too-alyssa-milano-I 508I 73882-htmlstory.html

Earl, J., Martin,A., McCarthy, J., y Sarah, S. (2004). The use of newspaper data in the study of collective action. Annual Review of Sociology, núm. 30, pp. 65-80. doi: https://doi. org/I0.I I 46/annurev.soc.30.0I2703.II 0603

El Universal. (2019, 10 de abril). Acoso: ¿Sirve denunciar? El Universal. Recuperado de https://www.eluniversal.com. $\mathrm{mx} /$ articulo/editorial-el-universal/nacion/acoso-sirve-denunciar

Eyerman, R. (20I I). The Cultural Sociology of Political Assassination: From MLK and RFK to Fortuyn andVan Gogh. Londres: Palgrave MacMillan.

Folbre, N. (2020). Cooperation \& Conflict in the Patriarchal Labyrinth. Doedalus. The Journal of the American Academy of Arts \& Science, 149(1): 198-212. doi: https://doi. org/I0.1162/daed_a_0I782

Frías, S. (2019). Hostigamiento y acoso sexual. El caso de una institución de procuración de justicia. Estudios 
Sociológicos de El Colegio de México, 38(I I 2): 103-139. doi: I0.2420I/es.2020v38n I I2.1745

Fuentes,A. (2019,3I de marzo). Más que un hashtag, un grito de desesperación. El Universal. Recuperado de https:// www.eluniversal.com.mx/articulo/angelica-fuentes/ cartera/mas-que-un-hashtag-un-grito-de-desesperacion Gamés, G. (2019, 8 de abril). Capturar y someter. Milenio. Recuperado de https://www.milenio.com/opinion/gilgames/uno-hasta-el-fondo/capturar-y-someter

García, S. (2019, 7 de abril). \#MeToo. Excélsior. Recuperado de https://www.excelsior.com.mx/opinion/opinion-del-experto-nacional/metoo// 306246

Geertz, C. (2003). La interpretación de las culturas. Barcelona/ México.

Guarneros, F. (2019, 3 I de marzo).\#MeToo. Excélsior. Recuperado de https://www.excelsior.com.mx/opinion/ fabiola-guarneros-saavedra/metoo/ / 304864

Gupta,A. (20I5). Fronteras borrosas: El discurso de la corrupción, la cultura de la política y el Estado imaginado. En P. Abrams, A. Gupta y T. Mitchell (eds.), Antropología del Estado (pp. 7I-I44). México: Fondo de Cultura Económica.

Hasunuma, L., y Ki-young, S. (2019). \#MeToo in Japan and South Korea: \#WeToo, \#WithYou. Journal of Women, Politics \& Policy, 40(I): 97-II I. doi: https://doi.org/I0.10 80/I554477X.2019.1563416

Hearn, J. (20I8). You, them, us, we, too?... online-offline, individual-collective, forgotten-remembered, harassment-violence. European Journal of Women's Studies, 25(2): 228-235. doi: I0. I I77/|3505068|8765286

Holland, K.,y Cortina, L. (20I3).When Sexism and Feminism Collide: The Sexual Harassment of Feminist Working Women. Psychology of Women Quarterly, 37(2): 192-208. doi: 10.1 | 77/ $/ 036$ | 6843 | 3482873 
Bibliografía

Hubert, L. (20I8). Is Metoo only a social movement or a legal movement too? Employee Rights and Employment Policy Journal, 22(2): 32I-336. doi: http://dx.doi.org//0.2139/ ssrn.3236309

Instituto Nacional de Estadística, Geografía e Informática (INEGI). (2016). Encuesta Nacional sobre la Dinámica de las Relaciones en los Hogares. Recuperado de https://www. inegi.org.mx/programas/endireh/2016/

Instituto Nacional de las Mujeres (Inmujeres). (2017). Informe estadístico de registro de casos de hostigamiento sexual y acoso sexual en la administración pública federal. Recuperado de https://www.gob.mx/cms/uploads/attachment/ file/403545/Informe_Casos_Hostigamiento.pdf

Instituto Nacional de las Mujeres, Secretaría de la Función Pública, y Comisión Nacional para Prevenir y Erradicar la Violencia Contra las Mujeres. (2018). Informe estadístico de registro de casos de hostigamiento sexual y acoso sexual en la administración pública federal 2018. Recuperado de https://www.gob.mx/cms/uploads/attachment/ file/506540/Informe_General_de_casos_vf.pdf Jonge,A. (2018). Corporate Social Responsibility through a Feminist Lens: Domestic Violence and the Workplace in the 2 Ist Century. Journal of Business Ethics, 148(3): 47I-487. doi: I0.1007/s I055 I-0 I5-30 I0-9

La Jornada. (2019, 2 de abril). Dilemas del \#MeToo. La Jornada. Recuperado de https://www.jornada.com. $\mathrm{mx} / 2019 / 04 / 02 /$ opinion/002a ledi

Laguna, M. (2020). The male body politic: Straight strippers, gay spaces, and queer masculinities. Sexuality, Gender \& Policy, I0(2): I - I2. doi: 10.1002/sgp2.120I5

Lamas, M. (2019, 5 de abril). Dilemas acerca del acoso. Reforma. Recuperado de https://www.reforma.com/ aplicaciones/editoriales/editorial.aspx?id= | 5407 |

\section{8}


Lavín, M. (2019, I 3 de abril). Preguntas y jacarandas. El UniBibliografía versal. Recuperado de https://www.eluniversal.com.mx/ columna/monica-lavin/cultura/preguntas-y-jacarandas

Loaeza, G. (2019, 9 de abril). \#YoTambién. Reforma. Recuperado de https://www.reforma.com/aplicaciones/ editoriales/editorial.aspx? id= 154285

Luengo, M. (20I8). Shaping solidarity in Argentina:The power of the civil sphere in repairing violence against women. En J. Alexander, y C. Tognato (eds.), The Civil Sphere in Latin America (pp. 39-65). Cambridge: Cambridge University Press.

Malvido, A. (2019, 17 de abril). \#MeToo, ética, arte y testosterona. El Universal. Recuperado de https://www. eluniversal.com.mx/columna/adriana-malvido/cultura/ metoo-etica-arte-y-testosterona

Martínez,J.(2019,7 de abril). La hoguera. Milenio. Recuperado de https://www.milenio.com/opinion/jose-luis-martinez/ el-santo-oficio/la-hoguera

Maza,V. (2019, 30 de marzo). Reflexiones sobre el \#MeToo. Milenio. Recuperado de https://www.milenio.com/ opinion/veronica-maza-bustamante/el-sexodromo/ reflexiones-sobre-el-metoo

McCarthy, J., McPhail., C., y Smith, J. ( 1996). Images of protest: Dimensions of selection bias in media coverage of Washington demonstrations, 1982 and 1991. American Sociological Review, 6 I (3): 478-499.

McLaughlin, H., Uggen, C., y Blackstone, A. (20I2). Sexual Harassment, Workplace Authority, and the Paradox of Power. American Sociological Review, 77(4): 625-647. doi: I0.1 I77/0003 I22412451728

McLean, J. (2020). Changing Digital Geographies, Changing Digital Geographies. Nueva York: Palgrave Macmillan. doi: 10.1007/978-3-030-28307-0

Monreal, C. (2019, 8 de abril). \#MeToo: Ni linchamiento ni revictimización. Excélsior. Recuperado de https://www. 
excelsior.com.mx/opinion/opinion-del-experto-nacional/metoo-ni-linchamiento-ni-revictimizacion/ / 30638 | Mueller, C., Mulingue, M., y Glass, J. (2002). Interactional Processes and GenderWorkplace Inequalities. Social Psychology Quarterly, 65(2): I63- I85. doi: I0.2307/3090099 Musacchio, H. (2019, 4 de abril). El acoso a ArmandoVega-Gil. Excélsior. Recuperado de https://www.excelsior.com.mx/ opinion/humberto-musacchio/el-acoso-a-armando-vega-gil// 30570 I

Nicolás-Gavilán, M. T., Baptista-Lucio, M. P., y Padilla-Lavin, M.A. (2019). Effects of the \#MeToo campaign in media, social and political spheres:The case of Mexico. Interactions: Studies in Communication \& Culture, I0(3):273-290. doi: 10.1386/iscc.10.3.273_I

O'Brien, M. (2020). Women's voices get louder: Sexual harassment on our screens and in our workplaces. Alternative Law Journal, 45(2): |47-I48. doi: 10.1 I77/1037969X20927822

Ohlheiser, A. (20I7). The woman behind "Me Too" knew the power of the phrase when she created it - 10 years ago. Los Angeles Times. Recuperado de. https://web. archive.org/web/20171019201825/https:/www.washingtonpost.com/news/the-intersect/wp/2017/10/19/ the-woman-behind-me-too-knew-the-power-of-thephrase-when-she-created-it-10-years-ago/?utm_term=. bc28f88I 5787

Ortiz, D., Myers, D., Walls, E., y Díaz, M. (2005). Where do we stand with newspaper data? Mobilization: An International Quarterly, 10(3): 397-4I9. doi: 10.I78I3/ maiq. 10.3.8360r760k3277t42

Peña, S., Larrondo,A., y Agirreazkuenaga, I. (2020). Etiquetar el feminismo. Análisis de los cibermedios iberoamericanos. Cuadernos.info, núm. 46, pp. 47-65. doi: I0.7764/ cdi.46. 1456 
Peñaloza, P. (2019a, 29 de marzo). Ruta sonora. La JorBibliografía nada. Recuperado de https://www.jornada.com. $\mathrm{mx} / 20$ l 9/03/29/opinion/a08olesp

—_. (2019b, 5 de abril). Ruta sonora. Por un \#MeTooMúsicosMexicanos menos irresponsable. La Jornada. Recuperado de https://www.jornada.com.mx/2019/04/05/ opinion/al0olesp

Perrott, T. (20/9). Methodological Awareness in Feminist Research: Reclaiming Experiences of Hostility in Workplace Studies, International Journal of Qualitative Methods, I8(I): I-9. doi: I0.I I77/|6094069|9854836

Petrich, B. (2019, 3 de abril). El \#MeToo mexicano, hoguera fuera de control.La Jornada. Recuperado de https://www. jornada.com.mx/2019/04/03/opinion/0 I7a I pol

Poniatowska, E. (20 I 9, 4 de abril). Denuncias anónimas indignantes. La Jornada. Recuperado de https://www.jornada. com.mx/2019/04/04/opinion/a04a l esp

Revueltas, R. (2019, 7 de abril). El feminismo confiscado. Milenio. Recuperado de https://www.milenio.com/ opinion/roman-revueltas-retes/la-semana-de-roman-revueltas-retes/el-feminismo-confiscado

Rhode, D. (2019). \#MeToo: Why Now? What Next? Duke Law Journal, 69(2): 377-428. Recuperado de https://scholarship.law.duke.edu/dlj/vol69/ iss $2 / 3$

Río, M. (2008). Usos y abusos de la prensa como fuente de datos sobre las acciones colectivas. Revista de Metodología de Ciencias Sociales, 16(2): 59-84. doi: https://doi.org/10.5944/empiria.16.2008.1390

Rivera, C. (2020). Women against the Feminicide Machine in Mexico. World Literature Today, 94(1): 50-54. doi: 10.11693/hyhz20181000233

Rocha, R. (2019, 3 de abril). ¿No se culpe a nadie de su muerte? El Universal. Recuperado de https://www.elu- 
niversal.com.mx/articulo/ricardo-rocha/nacion/no-seculpe-nadie-de-su-muerte

Rodríguez, L., Barraza, D., Salazar, J.,yVargas, R. (20।9). Index of suicide risk in Mexico using Twitter. Journal of Social Researches, 5(I5): I- I 3. doi: I0.35429/jsr.20 I 9.I5.5.I.I3 Rojas, P. (2019, 8 de abril). Del acoso secreto a la denuncia anónima. El Universal. Recuperado de https://www.eluniversal.com.mx/columna/paola-rojas/nacion/del-acoso-secreto-la-denuncia-anonima

Sánchez, M. (2020). Las políticas públicas de igualdad de género en México. Revista Internacional de Derechos Humanos, IO(I): I75-2 I2. Recuperado de https://www. revistaidh.org/ojs/index.php/ridh/article/view/I55

Sarmiento, S. (2019, 2 de abril). No hay salida. Reforma. Recuperado de https://www.reforma.com/aplicaciones/ editoriales/editorial.aspx? id $=\mid 53866$

Sefchovich, S. (2019, 7 de abril). MeToo y otras justicias. El Universal. Recuperado de https://www.eluniversal.com. $\mathrm{mx} / \mathrm{articulo/sara-sefchovich/nacion/metoo-y-otras-}$ justicias

Sierra, Y. (2019a, 6 de abril). Cimbrar la tierra. Excélsior. Recuperado de https://www.excelsior.com.mx/opinion/ yuriria-sierra/cimbrar-la-tierra// 306103

—_. (2019b, 2 de abril). La culpa no es del \#MeToo. Excélsior. Recuperado de https://www.excelsior.com.mx/opinion/ yuriria-sierra/la-culpa-no-es-del-metoo/ / 3052 I 8

Smelser, N. (2004). Psychological and cultural trauma. En J. Alexander, R. Eyerman, B. Giesen, N. Smelser y P. Sztompka (eds.), Cultural Trauma and Collective Identity (pp. 3 I-59). Berkeley: University of California Press.

Sosa, M. (2019). Un \#MeToo frente al suicidio: Problematizar el caso mexicano, en XIII Jornadas de Sociología. Las cuestiones de la sociología y la sociología en cuestión. Facultad de Ciencias Sociales, Universidad de Buenos Aires, 26 al 30 de agosto de 2019. 
Taylor, M.,Vigil, H., y Santiago, M. (2018). \#MeToo and How Bibliografía It May Impact You Too Across Latin America. Labor Law Journal, 69(4): 242-244. Recuperado de http://ezproxy. lib.ucalgary.ca/login?url=http://search.ebscohost.com/ login.aspx?direct $=$ true \&db=bsh\&AN $=|33445| 63 \&$ site $=$ ehost-live

Tello, I. (2019, 5 de abril). \#MeToo y las deficiencias del sistema de justicia penal. El Universal. Recuperado de https://www.eluniversal.com.mx/articulo/irene-teIlo-arista/nacion/metoo-y-las-deficiencias-del-sistema-de-justicia-penal

Ushma, N. (2019). When Scientists Say, Me Too. Scientific American. Recuperado de https://web.archive.org/ web/20 I 7 I0260542 I5/https:/blogs.scientificamerican. com/voices/when-scientists-say-me-too/

Vela, E. (20I9, 3I de marzo). \#MeToo. Reforma. Recuperado de https://www.reforma.com/aplicaciones/editoriales/ editorial.aspx?id=| 53730\&referer=--7d6|6|65662f3a3a6262623b727a7a7279703b767a783a

Villanueva, E. (2019, 3 de abril). Los alcances de \#MeToo. Excélsior. Recuperado de https://www.excelsior.com. $\mathrm{mx} /$ nacional/el-opinador-en-excelsior-digital-los-alcances-de-metoo/I 305566

Villeda, A. (2019,6 de abril). \#MeToo y la doble personalidad. Milenio. Recuperado de https://www.milenio.com/ opinion/alfredo-villeda/fusilerias/metoo-y-la-doble-personalidad

Virrueta, P.(2019, 3 de abril). La responsabilidad de un \#hashtag. Excélsior. Recuperado de https://www.excelsior.com. $\mathrm{mx/opinion/paola-virrueta/la-responsabilidad-de-un-}$ -hashtag/I 305234

Zamarrón, H. (2019a, 3I de marzo). El sexo es política, hablemos. Milenio. Recuperado de https://www.milenio. com/opinion/hector-zamarron/afinidades-selectivas/ el-sexo-es-politica-hablemos 
Bibliografía $\mid \longrightarrow$. (2019b, 7 de abril). El nuevo conservadurismo. Milenio. Recuperado de https://www.milenio.com/opinion/ hector-zamarron/afinidades-selectivas/el-nuevo-conservadurismo

Zuckermann, L. (2019, 2 de abril). Sobre el movimiento \#MeToo y el suicidio de Armando Vega. Excélsior. Recuperado de https://www.excelsior.com.mx/opinion/ leo-zuckermann/sobre-el-movimiento-metoo-y-el-suicidio-de-armando-vega// 3052 I 4 